\title{
BANACH SPACES WITH THE (CRP) AND DOMINATED OPERATORS ON C(K)
}

\author{
G. Emmanuele
}

\begin{abstract}
We characterize Banach spaces with the (CRP) by means of dominated operators on $\mathrm{C}(\mathrm{K})$, improving an old result by J.J. Uhl; hence we apply our main theorem to the study of Pietsch integral operators and to Dunford-Pettis operators.
\end{abstract}

1. In [8] J.J. Uhl showed the following result: "Let $K$ be a compact Hausdorff space and $E$ a Banach space with the Radon-Nikodym property. If $T: C(K) \rightarrow E$ is a dominated operator, then $T$ is compact". Now we improve his result to the case of Banach spaces with the (CRP), also showing a converse of this result; i.e. we characterize the Banach spaces with the (CRP) by means of compact dominated operators on $C(K)$. Hence, we apply our theorem to the study of compactness of Pietsch integral operators and to Dunford-Pettis operators.

2. This section is devoted to state the definitions we need as well as the main theorem of the paper. Throughout $K$ will denote a compact Hausdorff space and $E$ a Banach space.

Definition 1. $E$ is said to possess the compact range property, in symbols (CRP), if any $E$-valued countably additive measure $\mu$, defined on a $\sigma$-field of subsets of an arbitrary set $S$, with finite variation $|\mu|(S)$, has relatively compact range.

Note that if $E$ has the Radon-Nikodym property then it has the (CRP). Since any Banach space with the weak Radon-Nikodym property has the (CRP), it is easy to see that there exist Banach spaces with the (CRP) but without the Radon-Nikodym property. In passing we observe that if $E$ has the $\mu$-(CRP) (see [6] for this definition) for a non-atomic measure $\mu$ then it has the (CRP).

A dominated operator $T: C(K) \rightarrow E$ will be defined according to Definition 1 in [3], p. 379 .

Work performed under the auspices of G.N.A.F.A. of C.N.R. and partially supported by M.U.R.S.T. of Italy (40\% Analisi Funzionale 1987).

doi:10.5186/aasfm.1991.1608 
Definition 2. An operator $T: C(K) \rightarrow E$ is called a dominated operator if there exists a countably additive regular positive Borel measure $\mu$ such that

$$
\|T(f)\| \leq \int_{K}|f(s)| d \mu, \quad f \in C(K) .
$$
(CRP).

Our main result is the following characterization of Banach spaces with the

Theorem 1. $E$ has the (CRP) property if and only if, for each $K$, any dominated operator $T: C(K) \rightarrow E$ is compact.

Proof. Assume $E$ has the (CRP) and $T: C(K) \rightarrow E$ is a dominated operator. It is well known ([3], p. 380) that there exists an $E$-valued countably additive regular Borel measure $\nu$ with finite variation $|\nu|$ such that

$$
T(f)=\int_{K} f(s) d \nu, \quad f \in C(K),
$$

(the integral is that defined in [3]; see also [2], p. 5). For a finite partition $\pi$ of $S$ in pairwise disjoint subsets from $\Sigma$ consider the $E$-valued measure

$$
\nu_{\pi}(A)=\sum_{x \in \pi} \frac{\nu(X)}{|\nu|(X)}|\nu|(X \cap A)
$$

and then the operator $T_{\pi}: C(K) \rightarrow E$ defined by

$$
T_{\pi}(f)=\int_{K} f(s) d \nu_{\pi}, \quad f \in C(K) .
$$

It is well known that for any $f \in C(K)$ we have

$$
\left\|T_{\pi}(f)-T(f)\right\| \leq\|f\|\left\|\nu_{\pi}-\nu\right\|(K)
$$

where $\left\|\nu_{\pi}-\nu\right\|(K)$ denotes the semivariation of $\nu_{\pi}-\nu$. In [5] (see Theorem 4.1) it is shown that $\left\|\nu_{\pi}-\nu\right\|(K) \rightarrow 0$ since $\nu$ has relatively compact range; so

$$
\sup _{\|f\| \leq 1}\left\|T_{\pi}(f)-T(f)\right\| \rightarrow 0 .
$$

Hence $T$ is compact, as a uniform limit of compact operators (note that any $T_{\pi}$ has range in a finite dimensional Banach space). Conversely, let $S$ be a set and $\Sigma$ be a $\sigma$-field of subsets of $S$ on which an $E$-valued countably additive measure $\nu$ with finite variation is defined. By the Stone representation theorem ([3]) there exist a compact, Hausdorff space $K$ and an $E$-valued countably additive 
regular Borel measure $\mu$ with finite variation such that $\nu(\Sigma)=\mu(\mathrm{Bo}(K))$. Hence, it is enough to show that $\mu$ has relatively compact range. Define an operator $T: L^{1}(K, \operatorname{Bo}(K),|\mu|) \rightarrow E$ by putting

$$
T(f)=\int_{K} f(s) d \mu .
$$

Its restriction $T^{\prime}$ to $C(K)$ is, clearly, a dominated operator; so it is compact, by assumption. Using the Lusin theorem and Tiezte extension theorem we can easily show that

$$
\mu(\operatorname{Bo}(K))=\left\{T\left(\chi_{A}\right): A \in \operatorname{Bo}(K)\right\} \subset\left\{\overline{T^{\prime}(f): f \in C(K),\|f\| \leq 1}\right\} .
$$

Hence $\mu$ has relatively compact range. We are done.

3. In this section we are going to apply our main result to other classes of operators between Banach spaces. We need some more definitions.

Definition 3. An operator $T: F \rightarrow E, F$ a Banach space, is called an absolutely summing operator if it maps weakly unconditionally converging series into absolutely summing ones.

Definition 4. An operator $T: F \rightarrow E$ is called a Pietsch integral operator if there exists an $E$-valued countably additive measure $\mu$ of bounded variation defined on the Borel (for the weak ${ }^{*}$ topology) sets of the unit ball $B\left(F^{*}\right)$ of $F^{*}$ such that for each $x \in F$

$$
T(x)=\int_{B\left(F^{*}\right)} x^{*}(x) d \mu
$$

Definition 5. An operator $T: F \rightarrow E$ is called a Dunford-Pettis operator if it maps weak Cauchy sequences into norm convergent ones.

It is well known that an operator $T: C(K) \rightarrow E$ is dominated if and only if it is absolutely summing if and only if it is Pietsch integral ([2] Chapter VI). Using Theorem 1 and some results about Pietsch integral operators we are able to prove the following result.

Theorem 2. $E$ has the (CRP) if and only if any Pietsch integral operator $T: F \rightarrow E$ is compact.

Proof. Assume that any Pietsch integral operator from $F$ into $E$ is compact and take $F=C(K)$. Theorem 1 gives the conclusion. Conversely, let $T: F \rightarrow E$ 
be a Pietsch integral operator. It is well known ([2], Chapter VI) that it has a factorization of the following type

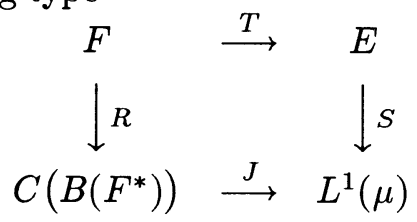

where $\mu$ is a suitable nonnegative regular Borel measure on $B\left(F^{*}\right)$ and $S$ is an operator with $\|S\| \leq 1$. Hence $S \cdot J$ is a dominated operator that is compact if one assumes that $E$ has the (CRP), by virtue of Theorem 1 . We are done.

In [2], Chapter VI, even nuclear operators were considered; it was shown that they are compact Pietsch integral operators and that if $E$ does not have the Radon-Nikodym property there exists an absolutely summing (i.e. Pietsch integral) operator from $C([0,1])$ into $E$ that is not nuclear. Combining this fact with our results we have the following consequence

Corollary 3. Let $E$ have the (CRP) but not the Radon-Nikodym property. Then there exists a compact Pietsch integral operator that is not nuclear, from $C([0,1])$ into $E$.

The final part of the note is devoted to Dunford-Pettis operators on $L^{1}$-spaces on finite measure spaces. We are able to prove the following result

Theorem 4. A Banach space $E$ has the (CRP) if and only if the operator $T: L^{1} \rightarrow E$ is Dunford-Pettis.

Proof. Assume any $T$ on a $L^{1}$-space is Dunford-Pettis and consider a dominated operator $T: C(K) \rightarrow E$. Such an operator factorizes through an $L^{1}$-space as observed before because it is Pietsch integral, in the following way

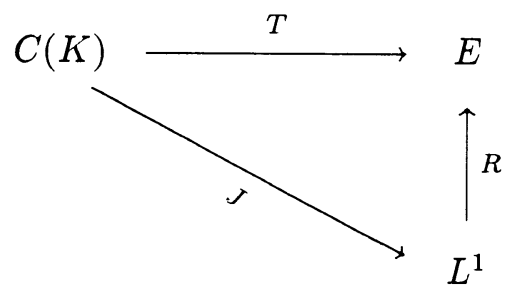

where $J$ is the canonical injection. $J$ maps bounded sets into relatively weakly compact sets and $R$ is Dunford-Pettis; hence $T$ is compact. An appeal to Theorem 1 gives our thesis. Conversely, assume $E$ has the (CRP) and consider an operator $T: L^{1} \rightarrow E$. Let $i_{\infty}$ be the canonical inclusion of $L^{\infty}$ into $L^{1}$. By using a proof like that one of Proposition 1 in [1] we can prove that $T$ is Dunford-Pettis if and only if $T \cdot i_{\infty}$ is compact. But $L^{\infty}$ is isomorphic to a suitable $C(K)$; let $j$ be the isomorphism. The operator $T \cdot i_{\infty} \cdot j$ is absolutely summing, hence dominated, because $i_{\infty}$ is (see [2], Chapter VI); then it is compact. Since $j$ is an isomorphism $T \cdot i_{\infty}$ has to be compact. We are done. 
As a consequence of Theorem 4 and the well known result by Pelczynski that a Banach space $E$ contains $l^{1}$ if and only if $E^{*}$ contains $L^{1}[0,1]$ ([7]) we have the following interesting corollary, which we only state:

Corollary 5. Let $E$ be a Banach space. Then $E$ does not contain $l^{1}$ if and only if $E^{*}$ has the (CRP), i.e. the (CRP) and the weak Radon-Nikodym property ([6]) coincide on dual Banach spaces.

Theorem 4 has even the following immediate consequence:

Corollary 6. Assume $X$ is a Banach space not containing a complemented copy of $l^{1}$ and $E$ a Banach space with the (CRP). Then any operator $T: X \rightarrow E$ that factorizes through an $L^{1}$-space is compact.

Proof. Let $T=R S$ be with $S: X \rightarrow L^{1}(\mu)$ and $R: L^{1}(\mu) \rightarrow E$. A result due to Bessaga-Pelczynski (see for instance [2], p. 88) now states that under our assumptions, $X^{*}$ cannot contain copies of $l^{\infty}$; hence $S^{*}: L^{\infty}(\mu) \rightarrow X^{*}$ is weakly compact (see Theorem 10 on p. 156 of [2]) and so even $S$ is. Since $R$ is DunfordPettis by Theorem 4, then $T$ is compact.

In the following corollary, we use the notion of local unconditional structure as introduced in [4].

Corollary 7. A Banach space $E$ has the (CRP) if and only if, for any Banach space $X$ with local unconditional structure and not containing complemented copies of $l^{1}$, any absolutely summing operator $T: X \rightarrow E$ is compact.

Proof. The "if"-part: Note that $C(K)$ spaces verify the hypotheses and that dominated operators on them are absolutely summing. The "only if" part: Note that an absolutely summing operator on a Banach space with local unconditional structure always factorizes through an $L^{1}$-space. 


\section{References}

[1] Bourgain, J.: Dunford-Pettis operators on $L^{1}$ and the Radon-Nikodym property. - Israel J. Math. 37, 1980, 34-47.

[2] Diestel, J., and J.J. UhL JR.: Vector measures. - Math. Surveys Monogr. 15, American Mathematical Society, 1977.

[3] Dinculeanu, N.: Vector measures. - Pergamon Press, 1967.

[4] Gordon, Y., and D.R. LEwis: Absolutely summing operators and local unconditional structure. - Acta Math. 133, 1974, 27-48.

[5] Graves, W.H., and W. Ruess: Compactness and weak compactness in spaces of compact range vector measures. - Canad. J. Math. 36, 1984, 1000-1020.

[6] Musial, K.: Martingales of Pettis integrable functions. - Proceedings of the conference "Measure theory", held in Oberwohlfach in 1979. Lecture Notes in Mathematics 794, Springer-Verlag, 1980, 324-329.

[7] Pelczynski, A.: On Banach spaces containing $L^{1}(\mu)$. - Studia Math. 30, 1968, 231-246.

[8] UHL, J.J., JR.: The compactness of certain dominated operators. - Rev. Roumaine Math. Pures Appl. 14, 1969, 1635-1637.

University of Catania

Department of Mathematics

Viale A. Doria 6

I-95125 Catania

Italy

Received 30 August 1990 